\title{
Precise Wettability Characterization of Carbonate Rocks to Evaluate Oil Recovery Using Surfactant-based Nanofluids
}

Foad Haeri and Dandina N. Rao

Craft and Hawkins Department of Petroleum Engineering, Louisiana State University, 3207

Patrick F. Taylor Hall, Baton Rouge, LA 70803

\section{Supporting Information}

Details of all the coreflood experiments of this study, including the core parameters, experimental conditions, oil recovery and pressure drop history-match, and relative permeability curves. 
Table 1. Initial parameters for waterflood using brine $(2 \mathrm{wt} . \% \mathrm{NaCl})$ at 500 psi and $72{ }^{\circ} \mathrm{F}$.

\begin{tabular}{|lll|}
\hline Core name: & \multicolumn{2}{l|}{ Indiana Limestone-10 (12X2) } \\
Porosity: & $18.14 \quad \%$ \\
Abs. Perm: & $23.59 \quad$ md \\
Pore Volume & $112 \quad$ cc \\
Oil & Yates crude oil \\
Brine (NaCl) & $2 \% \quad$ wt \\
Injection rate & 2 & $\mathrm{cc} / \mathrm{min}$ \\
Oil Recovery & $20.43 \quad \%$ \\
\hline
\end{tabular}

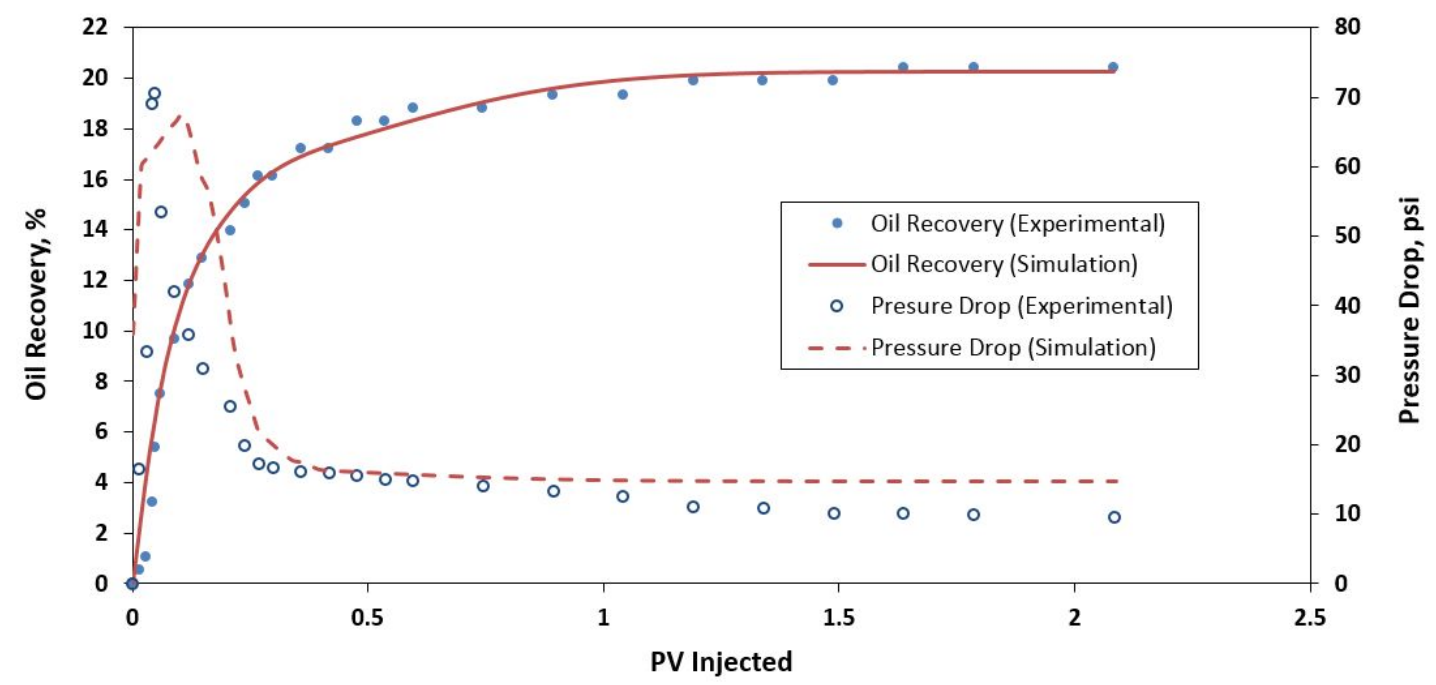

Figure 1. History match of oil recovery and pressure drop for waterflood ( $2 \mathrm{wt} . \% \mathrm{NaCl})$ at 500 psi and $72{ }^{\circ} \mathrm{F}$.

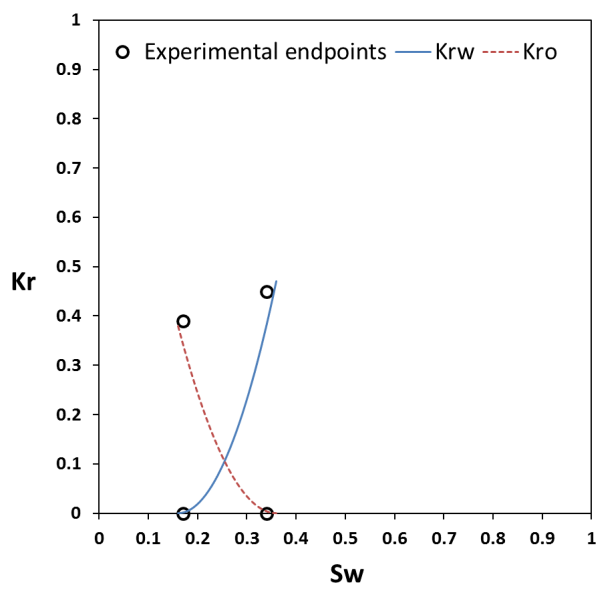

Figure 2. Relative permeability curves for waterflood $(2 \mathrm{wt} . \% \mathrm{NaCl})$ at $500 \mathrm{psi}$ and $72{ }^{\circ} \mathrm{F}$. 
Table 2. Initial parameters for coreflood using surfactant (ALF 13S, $2000 \mathrm{ppm}$ ) at $500 \mathrm{psi}$ and $72{ }^{\circ} \mathrm{F}$.

\begin{tabular}{|ll|}
\hline Core name: & \multicolumn{2}{l|}{ Indiana Limestone-15 (12X2) } \\
Porosity: & $16.03 \quad \%$ \\
Abs. Perm: & $26.24 \quad \mathrm{md}$ \\
Pore Volume & $99 \quad \mathrm{cc}$ \\
Oil & \multicolumn{2}{l|}{ Yates crude oil } \\
Brine (NaCl) & $2 \% \quad \mathrm{wt}$ \\
Surfactant & ALF $13 \mathrm{~S}(2000 \mathrm{ppm})$ \\
Injection rate & $2 \quad \mathrm{cc} / \mathrm{min}$ \\
Oil Recovery & $20.73 \quad \% \quad \mathrm{C}$ \\
\hline
\end{tabular}

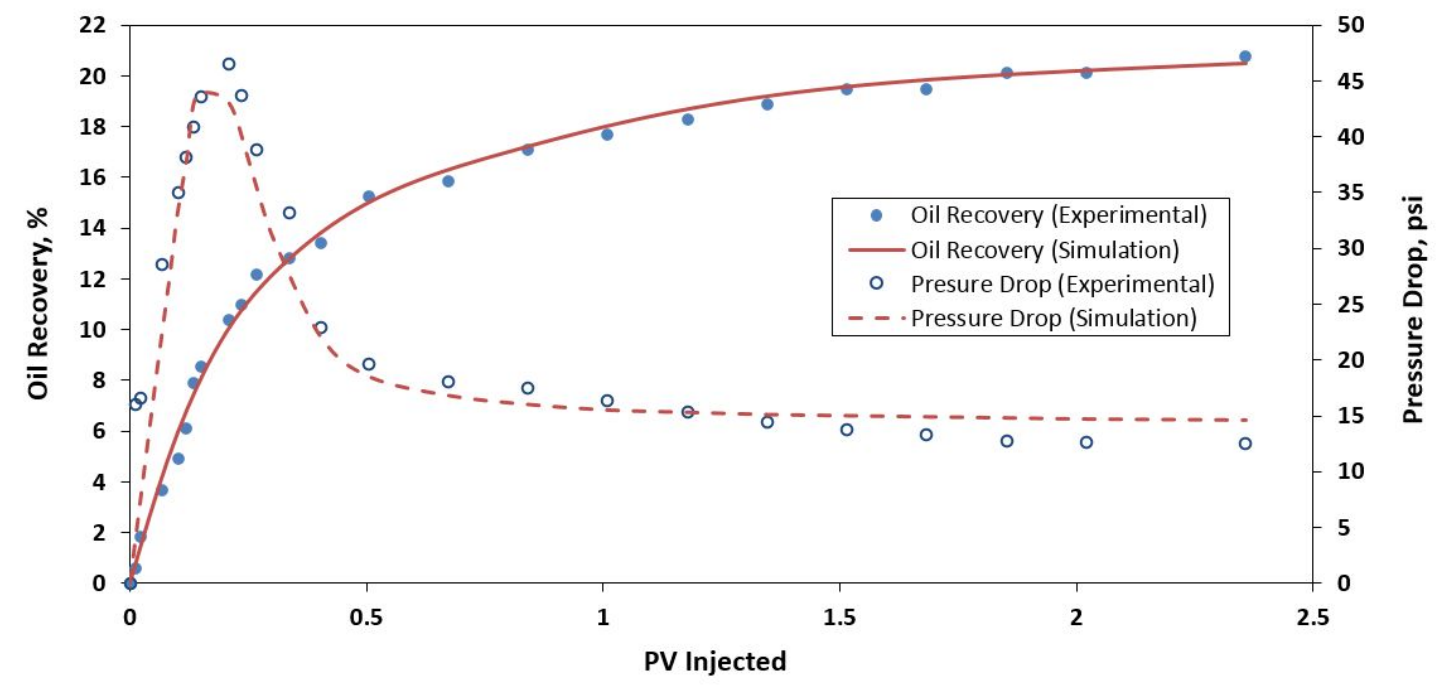

Figure 3. History match of oil recovery and pressure drop using surfactant (ALF 13S, $2000 \mathrm{ppm})$ at $500 \mathrm{psi}$ and $72^{\circ} \mathrm{F}$.

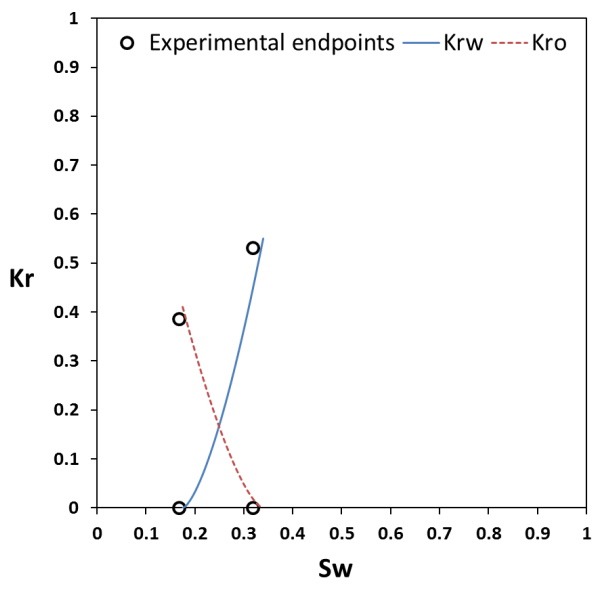

Figure 4. Relative permeability curves for coreflood using surfactant (ALF 13S, 2000 ppm) at 500 psi and $72{ }^{\circ} \mathrm{F}$. 
Table 3. Initial parameters for coreflood using brine-based nanofluid ( $0.4 \mathrm{wt} . \%$ NP in $2 \%$ $\mathrm{NaCl})$ at $500 \mathrm{psi}$ and $72^{\circ} \mathrm{F}$.

\begin{tabular}{|ll|}
\hline Core name: & \multicolumn{2}{l|}{ Indiana Limestone-14 (12X2) } \\
Porosity: & $14.73 \quad \%$ \\
Abs. Perm: & $18.59 \quad \mathrm{md}$ \\
Pore Volume & $91 \quad \mathrm{cc}$ \\
Oil & Yates crude oil \\
Brine (NaCl) & $2 \% \quad \mathrm{wt}$ \\
Nanofluid (brine-based) & $0.4 \mathrm{wt} . \% \mathrm{NP}$ \\
Injection rate & $2 \quad \mathrm{cc} / \mathrm{min}$ \\
Oil Recovery & $30.51 \quad \%$ \\
\hline
\end{tabular}

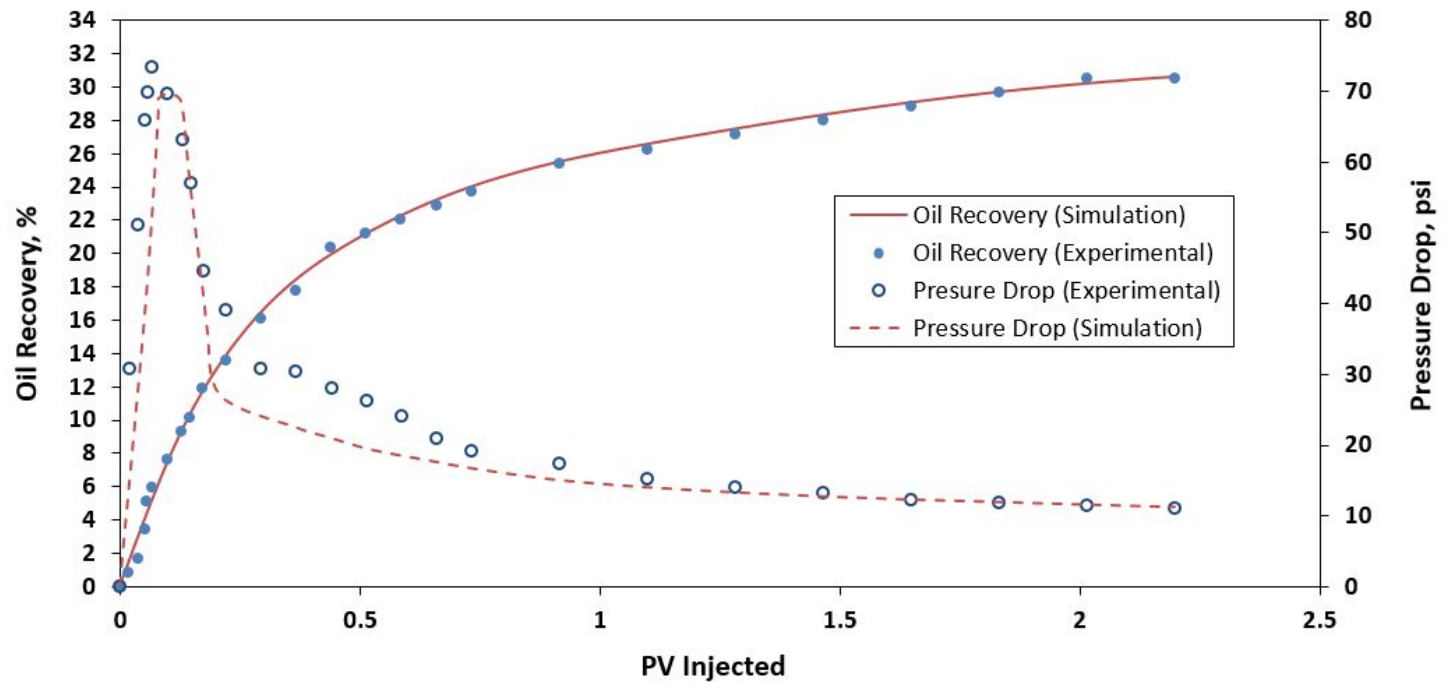

Figure 5. History match of oil recovery and pressure drop using brine-based nanofluid $(0.4$ wt. $\% \mathrm{NP}$ in $2 \% \mathrm{NaCl})$ at $500 \mathrm{psi}$ and $72{ }^{\circ} \mathrm{F}$.

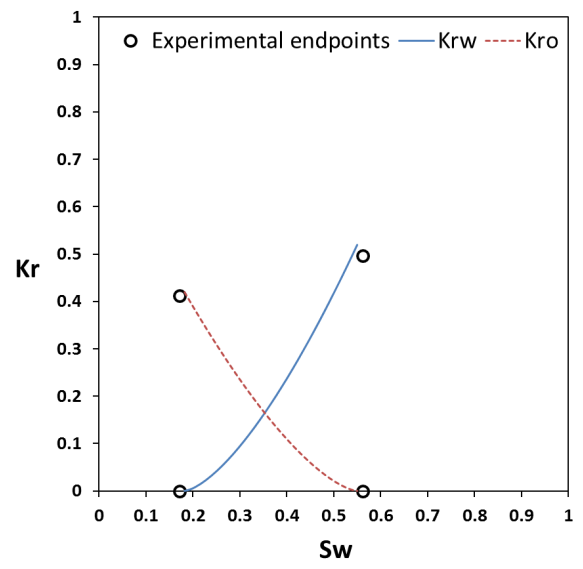

Figure 6. Relative permeability curves for coreflood using brine-based nanofluid ( $0.4 \mathrm{wt} . \%$ $\mathrm{NP}$ in $2 \% \mathrm{NaCl}$ ) at $500 \mathrm{psi}$ and $72{ }^{\circ} \mathrm{F}$. 
Table 4. Initial parameters for coreflood using surfactant-based nanofluid ( $0.4 \mathrm{wt} . \% \mathrm{NP}+$ ALF 13S, $2000 \mathrm{ppm}$ ) at $500 \mathrm{psi}$ and $72^{\circ} \mathrm{F}$.

\begin{tabular}{|lll|}
\hline Core name: & \multicolumn{2}{l|}{ Indiana Limestone-16 (12X2) } \\
Porosity: & $16.52 \quad \%$ \\
Abs. Perm: & $8.49 \quad \mathrm{md}$ \\
Pore Volume & $102 \quad \mathrm{cc}$ \\
Oil & Yates crude oil \\
Brine (NaCl) & $2 \% \quad \mathrm{wt}$ \\
Nanofluid (surfactant-based) & $0.4 \mathrm{wt} . \% \mathrm{NP}+$ ALF $13 \mathrm{~S}$ (2000 ppm) \\
Injection rate & $2 \quad \mathrm{cc} / \mathrm{min}$ \\
Oil Recovery & $57.33 \quad \%$ \\
\hline
\end{tabular}

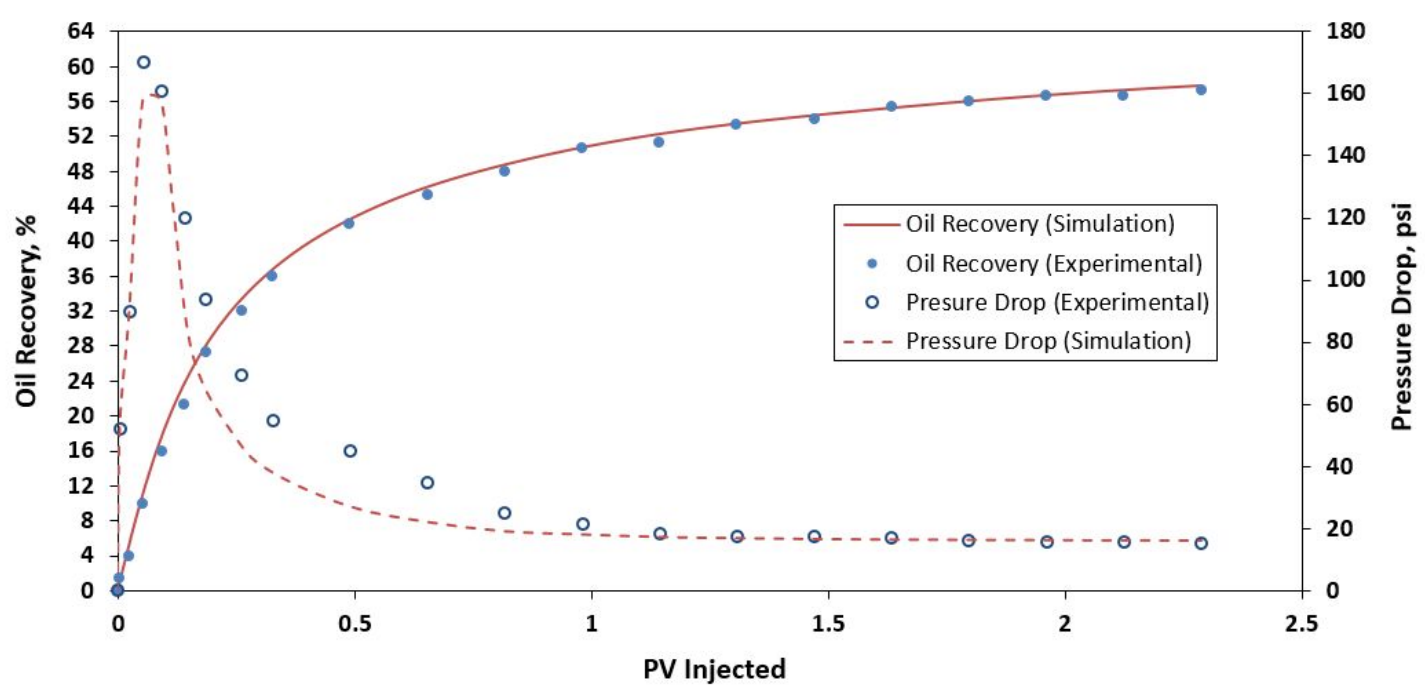

Figure 7. History match of oil recovery and pressure drop using surfactant-based nanofluid (0.4 wt.\% NP + ALF 13S, $2000 \mathrm{ppm})$ at $500 \mathrm{psi}$ and 72.

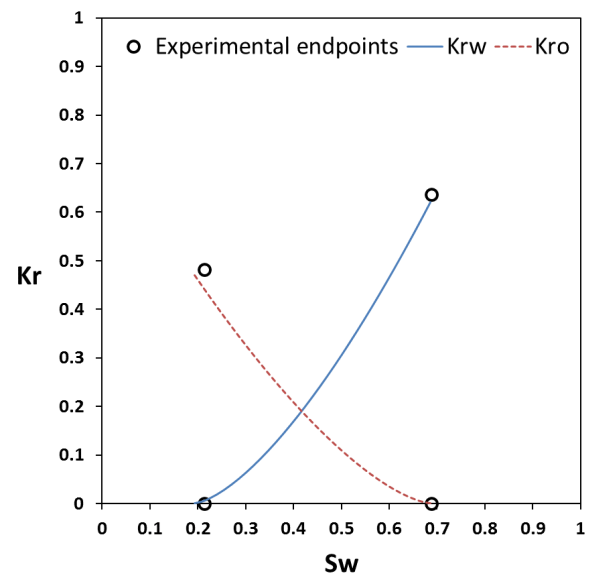

Figure 8. Relative permeability curves for coreflood using surfactant-based nanofluid (0.4 wt.\% NP + ALF 13S, $2000 \mathrm{ppm})$ at $500 \mathrm{psi}$ and $72^{\circ} \mathrm{F}$. 
Table 5. Initial parameters for coreflood using surfactant-based nanofluid ( $0.4 \mathrm{wt} . \% \mathrm{NP}+$ ALF $13 \mathrm{~S}, 1000 \mathrm{ppm})$ at $500 \mathrm{psi}$ and $72{ }^{\circ} \mathrm{F}$.

\begin{tabular}{|ll|}
\hline Core name: & \multicolumn{2}{l|}{ Indiana Limestone-17 (12X2) } \\
Porosity: & $17.17 \quad \%$ \\
Abs. Perm: & $15.81 \quad \mathrm{md}$ \\
Pore Volume & $106 \quad \mathrm{cc}$ \\
Oil & Yates crude oil \\
Brine (NaCl) & $2 \% \quad \mathrm{wt}$ \\
Nanofluid (surfactant-based) & $0.4 \mathrm{wt} . \% \mathrm{NP}+\mathrm{ALF}$ 13S (1000 ppm) \\
Injection rate & $2 \quad \mathrm{cc} / \mathrm{min}$ \\
Oil Recovery & $52.63 \quad \%$ \\
\hline
\end{tabular}

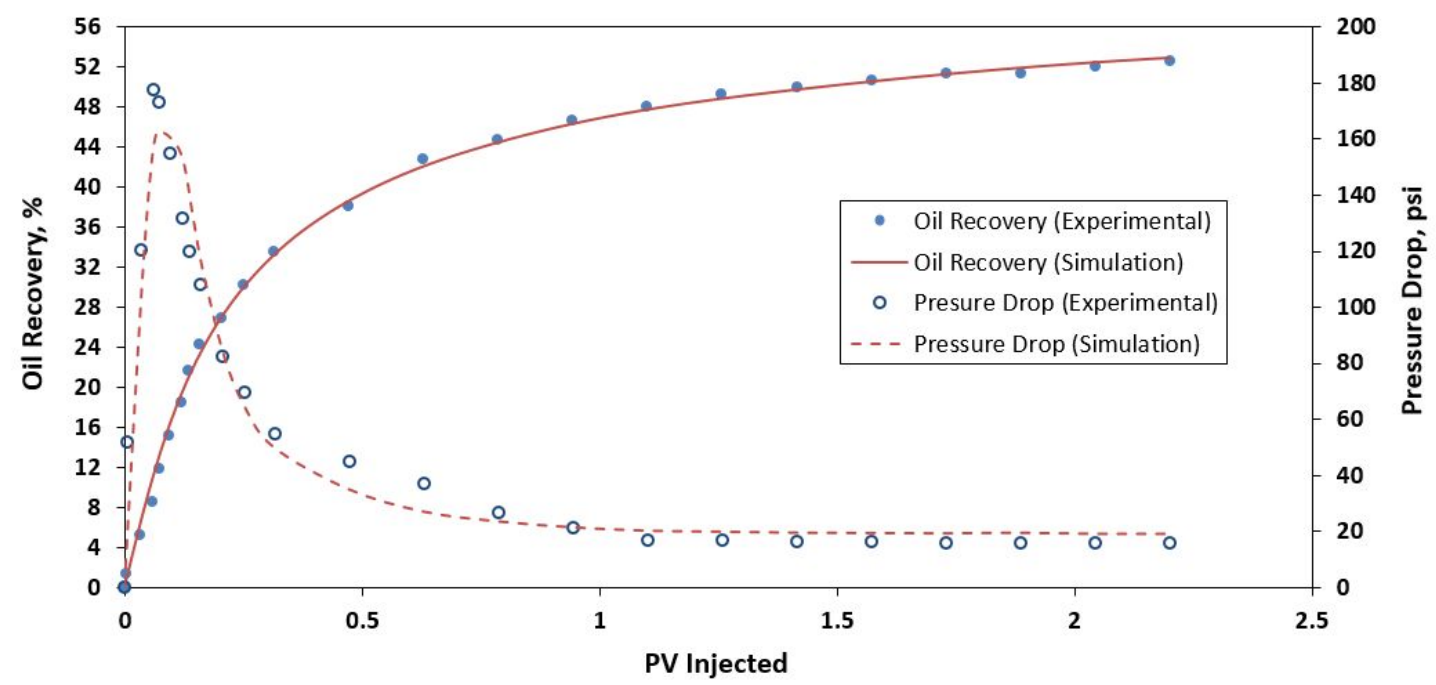

Figure 9. History match of oil recovery and pressure drop using surfactant-based nanofluid (0.4 wt.\% NP + ALF 13S, 1000 ppm) at 500 psi and 72.

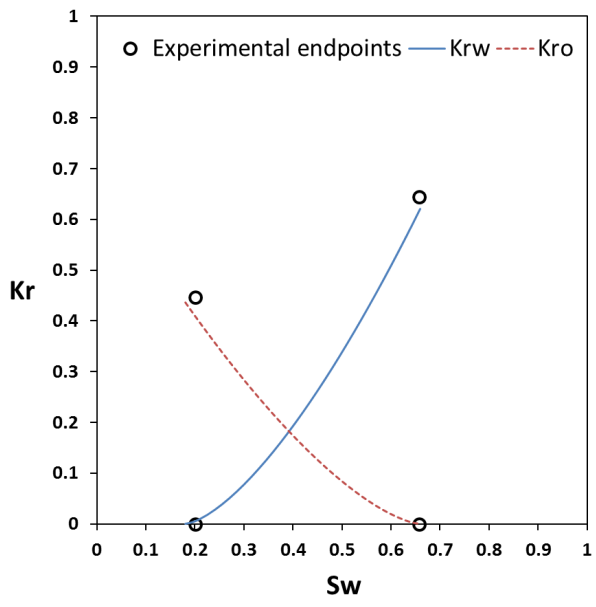

Figure 10. Relative permeability curves for coreflood using surfactant-based nanofluid (0.4 wt.\% NP + ALF 13S, $1000 \mathrm{ppm})$ at $500 \mathrm{psi}$ and $72{ }^{\circ} \mathrm{F}$. 
Table 6. Initial parameters for coreflood using surfactant (ALF 9S, $2000 \mathrm{ppm}$ ) at $500 \mathrm{psi}$ and $72^{\circ} \mathrm{F}$.

\begin{tabular}{|lll|}
\hline Core name: & \multicolumn{3}{l|}{ Indiana Limestone-X (12X2) } \\
Porosity: & 16.36 & $\%$ \\
Abs. Perm: & 17.95 & $\mathrm{md}$ \\
Pore Volume & 101 & $\mathrm{cC}$ \\
Oil & \multicolumn{2}{l|}{ Yates crude oil } \\
Brine (NaCl) & $2 \%$ & $\mathrm{wt}$ \\
Surfactant & ALF $9 \mathrm{~S}$ & $(2000 \mathrm{ppm})$ \\
Injection rate & 2 & $\mathrm{cc} / \mathrm{min}$ \\
Oil Recovery & 48.19 & $\%$ \\
\hline
\end{tabular}

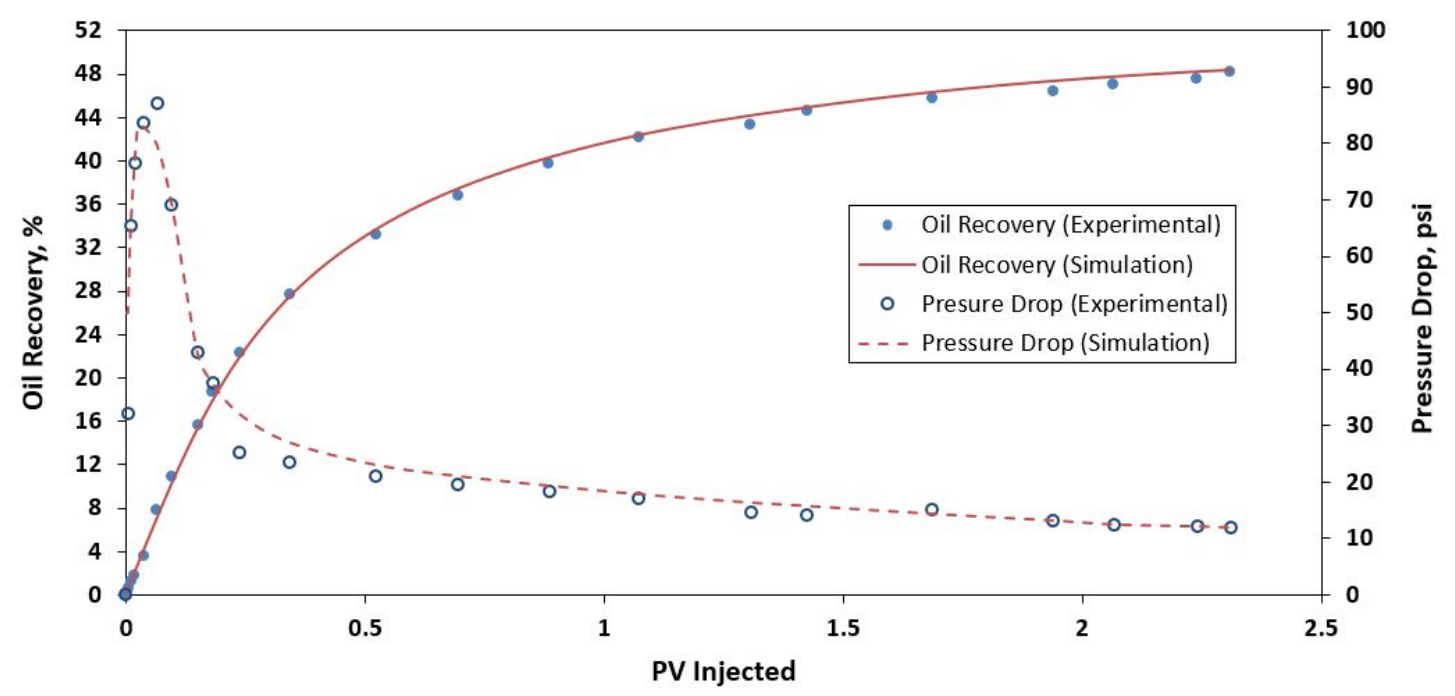

Figure 11. History match of oil recovery and pressure drop using surfactant (ALF 9S, $2000 \mathrm{ppm}$ ) at $500 \mathrm{psi}$ and $72^{\circ} \mathrm{F}$.

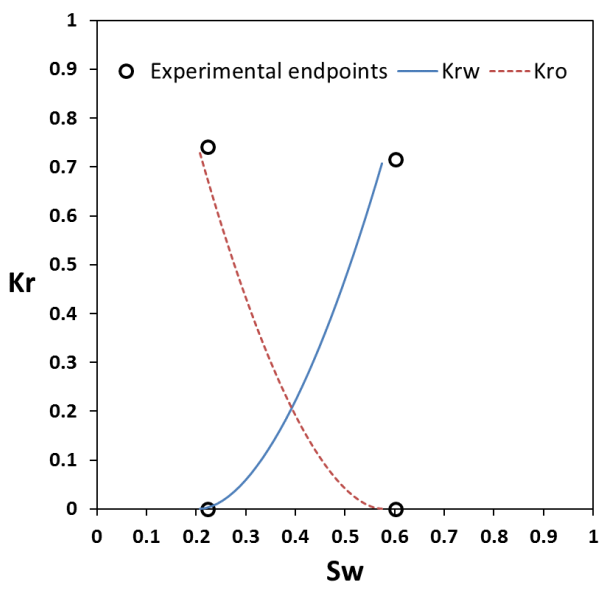

Figure 12. Relative permeability curves for coreflood using surfactant (ALF 9S, 2000 ppm) at 500 psi and $72{ }^{\circ} \mathrm{F}$. 
Table 7. Initial parameters for coreflood using surfactant-based nanofluid ( 0.4 wt. $\%$ $\mathrm{NP}+\mathrm{ALF} 9 \mathrm{~S}, 2000 \mathrm{ppm})$ at $500 \mathrm{psi}$ and $72{ }^{\circ} \mathrm{F}$.

\begin{tabular}{|c|c|c|}
\hline Core name: & \multicolumn{2}{|c|}{ Indiana Limestone-X (12X2) } \\
\hline Porosity: & 16.36 & $\%$ \\
\hline Abs. Perm: & 17.95 & $\mathrm{md}$ \\
\hline Pore Volume & 101 & $\mathrm{cc}$ \\
\hline Oil & \multicolumn{2}{|c|}{ Yates crude oil } \\
\hline Brine (NaCl) & $2 \%$ & wt \\
\hline Nanofluid (surfactant-based) & \multicolumn{2}{|c|}{0.4 wt.\% NP + ALF 9S (2000 ppm) } \\
\hline Injection rate & 2 & $\mathrm{cc} / \mathrm{min}$ \\
\hline Oil Recovery & 92.68 & $\%$ \\
\hline
\end{tabular}

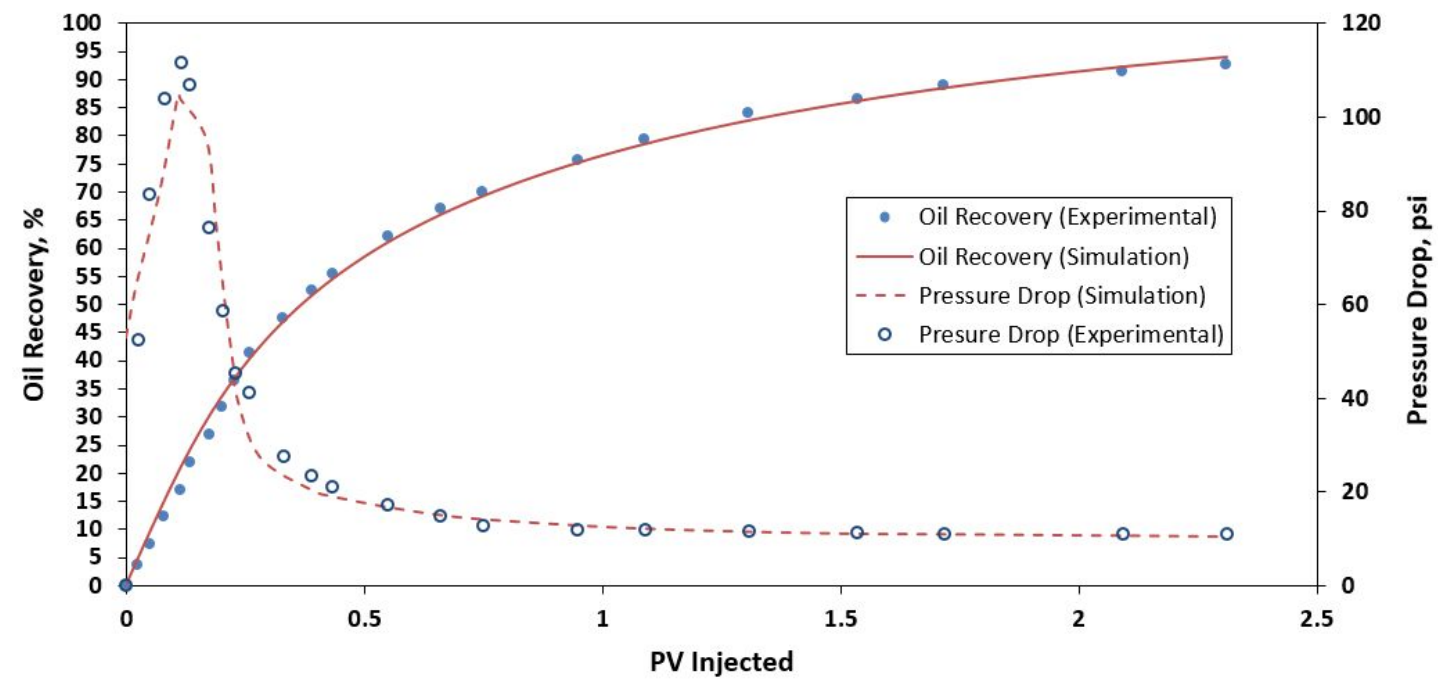

Figure 13. History match of oil recovery and pressure drop using surfactant-based nanofluid (0.4 wt.\% NP+ALF 9S, $2000 \mathrm{ppm})$ at $500 \mathrm{psi}$ and $72{ }^{\circ} \mathrm{F}$.

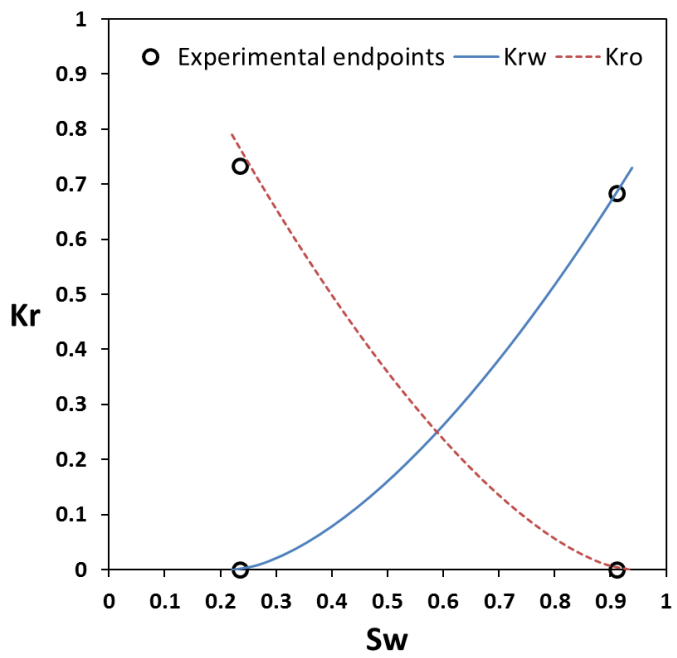

Figure 14. Relative permeability curves for coreflood using surfactant-based nanofluid (0.4 wt.\% NP+ALF 9S, $2000 \mathrm{ppm})$ at $500 \mathrm{psi}$ and $72^{\circ} \mathrm{F}$. 
Table 8. Initial parameters for coreflood using surfactant-based nanofluid ( $0.4 \mathrm{wt} . \%$ $\mathrm{NP}+\mathrm{ALF} 9 \mathrm{~S}, 1000 \mathrm{ppm})$ at $500 \mathrm{psi}$ and $72{ }^{\circ} \mathrm{F}$.

\begin{tabular}{|c|c|c|}
\hline Core name: & \multicolumn{2}{|c|}{ Indiana Limestone-X (12X2) } \\
\hline Porosity: & 16.36 & $\%$ \\
\hline Abs. Perm: & 17.95 & $\mathrm{md}$ \\
\hline Pore Volume & 101 & $\mathrm{cc}$ \\
\hline Oil & \multicolumn{2}{|c|}{ Yates crude oil } \\
\hline Brine (NaCl) & $2 \%$ & wt \\
\hline Nanofluid (surfactant-based) & \multicolumn{2}{|c|}{0.4 wt.\% NP + ALF 9S (1000 ppm) } \\
\hline Injection rate & 2 & $\mathrm{cc} / \mathrm{min}$ \\
\hline Oil Recovery & 86.25 & $\%$ \\
\hline
\end{tabular}

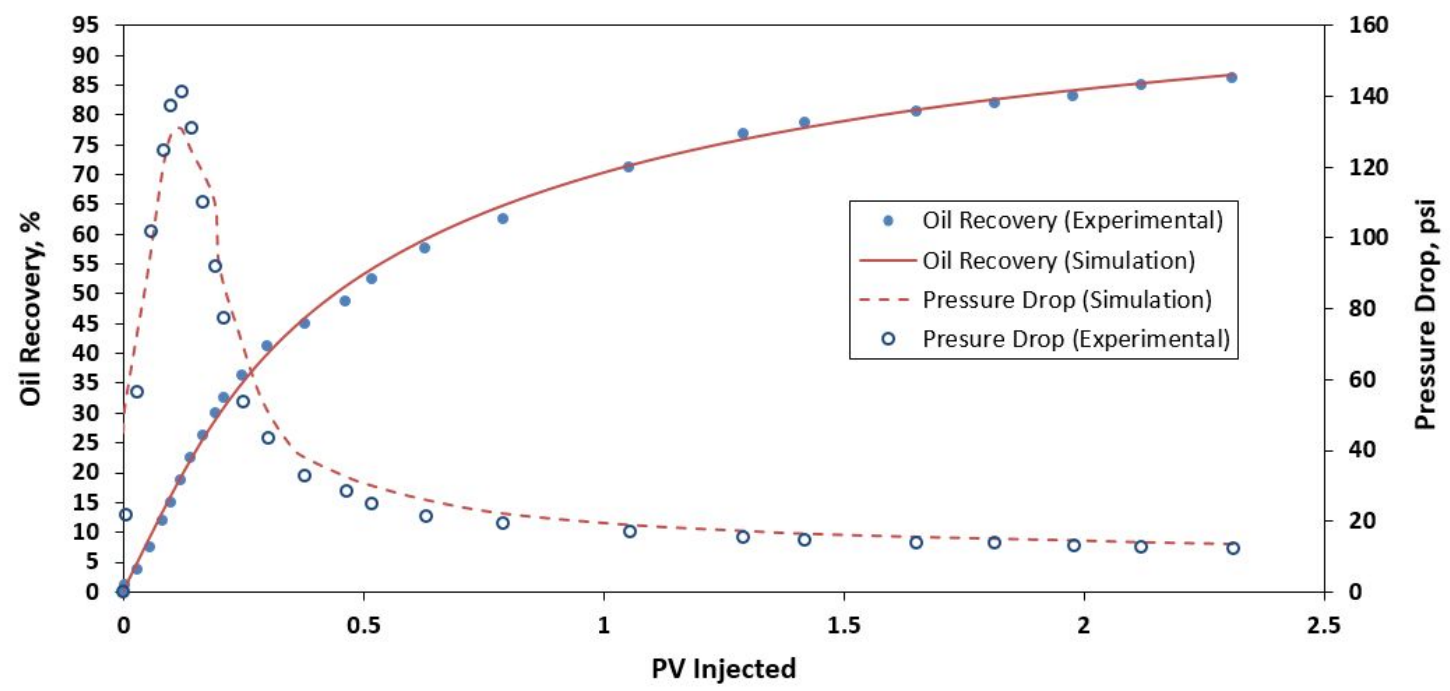

Figure 15. History match of oil recovery and pressure drop using surfactant-based nanofluid (0.4 wt.\% NP+ALF 9S, $1000 \mathrm{ppm})$ at $500 \mathrm{psi}$ and $72{ }^{\circ} \mathrm{F}$.

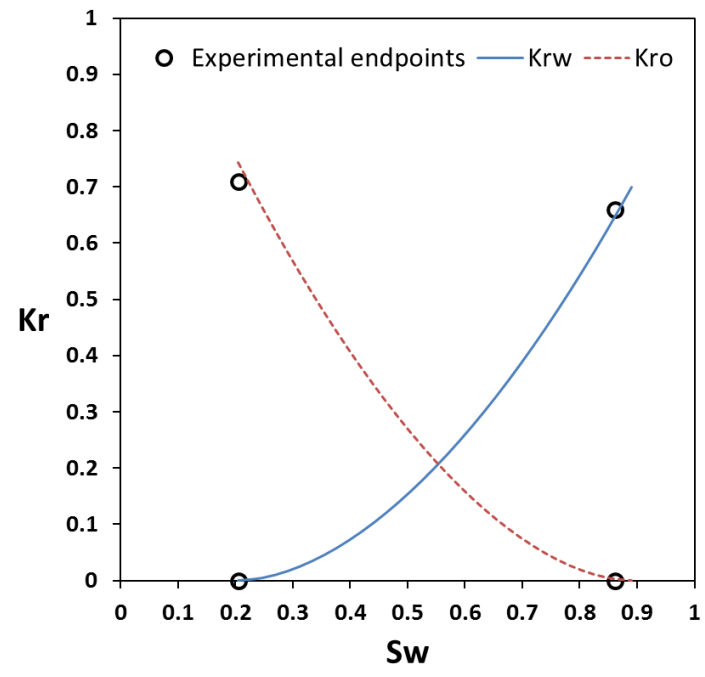

Figure 16. Relative permeability curves for coreflood using surfactant-based nanofluid (0.4 wt.\% NP+ALF 9S, $1000 \mathrm{ppm})$ at $500 \mathrm{psi}$ and $72{ }^{\circ} \mathrm{F}$. 
Table 9. Initial parameters for waterflood using brine $(2 \mathrm{wt} . \% \mathrm{NaCl})$ at reservoir conditions $\left(700\right.$ psi \& $\left.150{ }^{\circ} \mathrm{F}\right)$.

\begin{tabular}{|lll|}
\hline Core name: & \multicolumn{2}{l|}{ Indiana Limestone-12 (12X2) } \\
Porosity: & $16.35 \quad \%$ \\
Abs. Perm: & $14.87 \quad \mathrm{md}$ \\
Pore Volume & $101 \quad \mathrm{cc}$ \\
Oil & Yates crude oil \\
Brine (NaCl) & $2 \%$ & $\mathrm{wt}$ \\
Injection rate & 2 & $\mathrm{cc} / \mathrm{min}$ \\
Oil Recovery & 17.5 & $\%$ \\
\hline
\end{tabular}

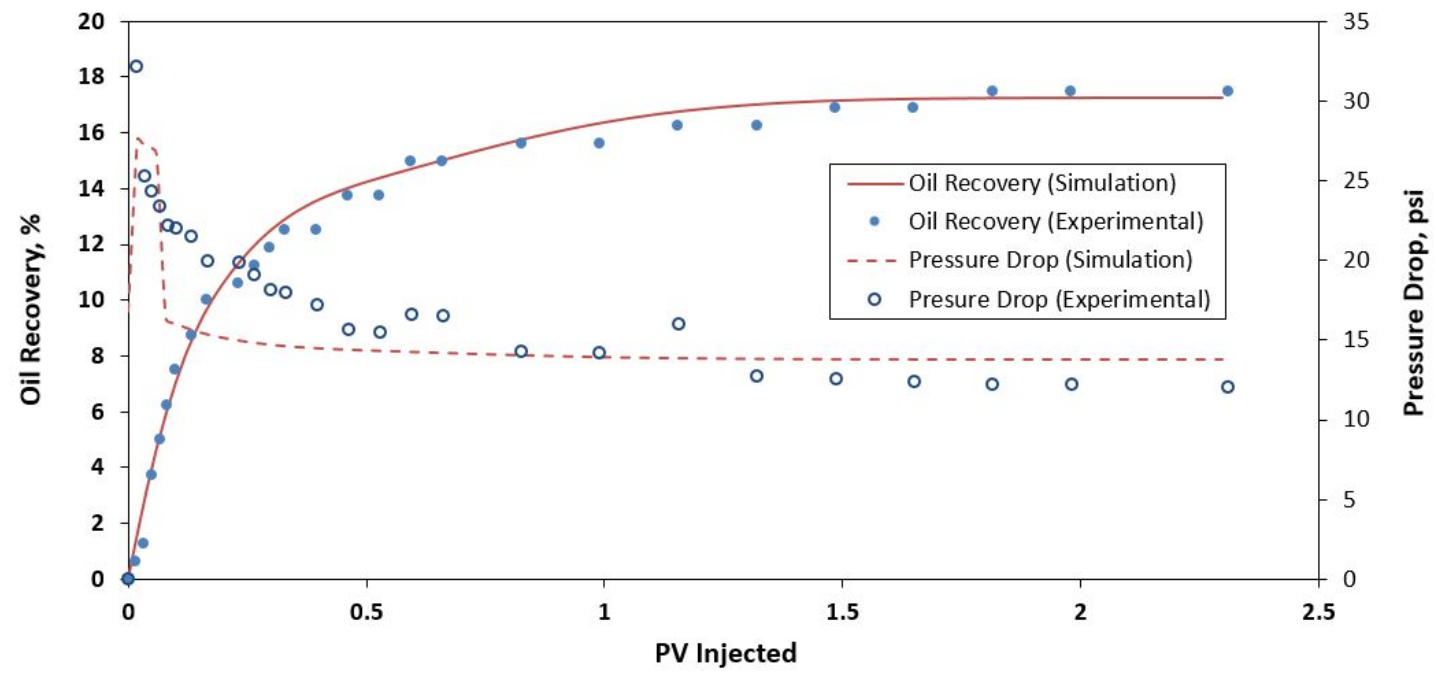

Figure 17. History match of oil recovery and pressure drop for waterflood $(2 \mathrm{wt} . \% \mathrm{NaCl})$ at reservoir conditions.

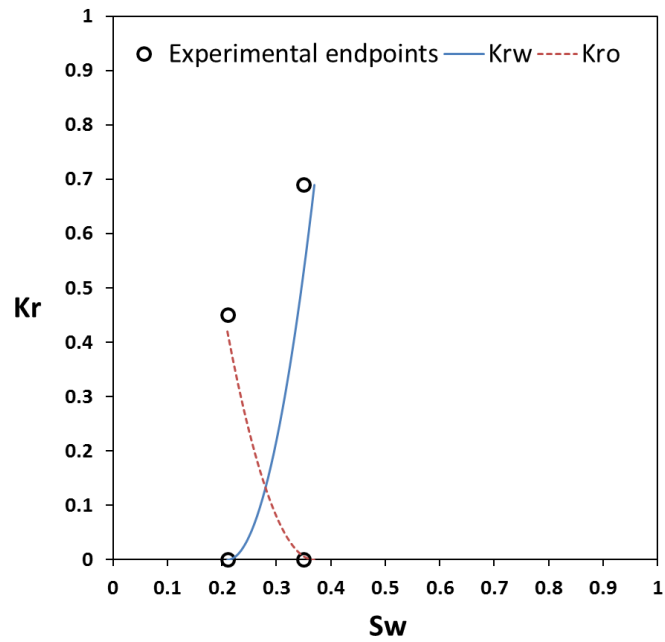

Figure 18. Relative permeability curves for waterflood $(2 \mathrm{wt} . \% \mathrm{NaCl})$ at reservoir conditions $\left(700 \mathrm{psi} \& 150{ }^{\circ} \mathrm{F}\right)$. 
Table 10. Initial parameters for coreflood using surfactant (SOL 938, $2000 \mathrm{ppm}$ ) at reservoir conditions $\left(700 \mathrm{psi} \& 150{ }^{\circ} \mathrm{F}\right)$.

\begin{tabular}{|ll|}
\hline Core name: & \multicolumn{2}{l|}{ Indiana Limestone-18 (12X2) } \\
Porosity: & $15.71 \quad \%$ \\
Abs. Perm: & $24.92 \quad \mathrm{md}$ \\
Pore Volume & $97 \quad \mathrm{cc}$ \\
Oil & Yates crude oil \\
Brine (NaCl) & $2 \% \quad \mathrm{wt}$ \\
Surfactant & SOL $938(2000 \mathrm{ppm})$ \\
Injection rate & $2 \quad \mathrm{cc} / \mathrm{min}$ \\
Oil Recovery & $21.52 \quad \%$ \\
\hline
\end{tabular}

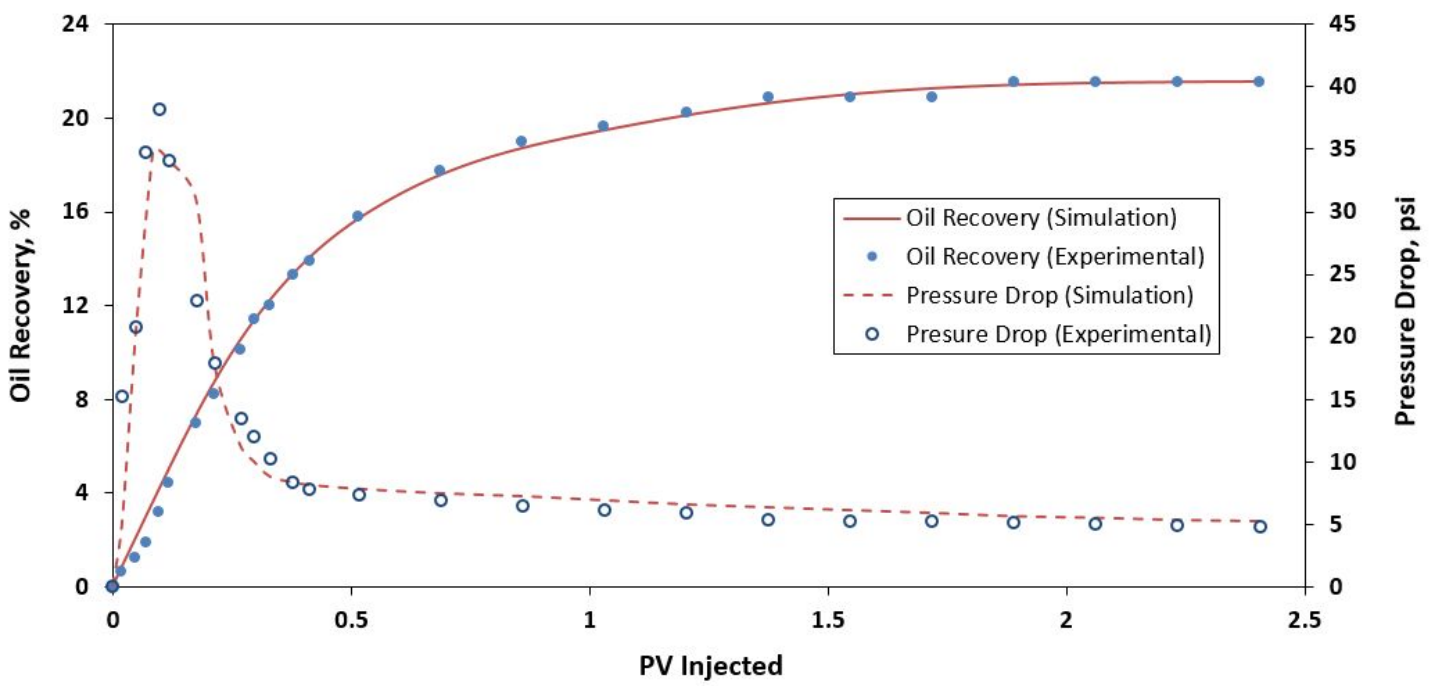

Figure 19. History match of oil recovery and pressure drop using surfactant (SOL 938, $2000 \mathrm{ppm})$ at reservoir conditions $\left(700 \mathrm{psi} \& 150^{\circ} \mathrm{F}\right)$.

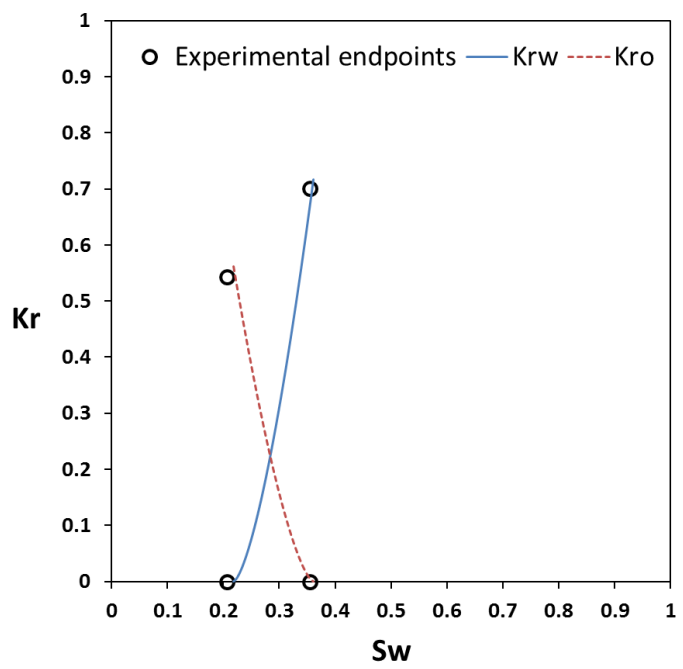

Figure 20. Relative permeability curves for coreflood using surfactant (SOL 938, 2000 ppm) at reservoir conditions $\left(700 \mathrm{psi} \& 150^{\circ} \mathrm{F}\right)$. 
Table 11. Initial parameters for coreflood using brine-based nanofluid ( 0.4 wt. $\%$ NP in $2 \% \mathrm{NaCl})$ at reservoir conditions $\left(700\right.$ psi \& $\left.150{ }^{\circ} \mathrm{F}\right)$.

\begin{tabular}{|ll|}
\hline Core name: & \multicolumn{2}{l|}{ Indiana Limestone-19 (12X2) } \\
Porosity: & $17.00 \quad \%$ \\
Abs. Perm: & $31.29 \quad \mathrm{md}$ \\
Pore Volume & $105 \quad \mathrm{cc}$ \\
Oil & Yates crude oil \\
Brine (NaCl) & $2 \% \quad \mathrm{wt}$ \\
Nanofluid (brine-based) & $0.4 \mathrm{wt} \% \mathrm{NP}$ \\
Injection rate & $2 \quad \mathrm{cc} / \mathrm{min}$ \\
Oil Recovery & $28.57 \quad \%$ \\
\hline
\end{tabular}

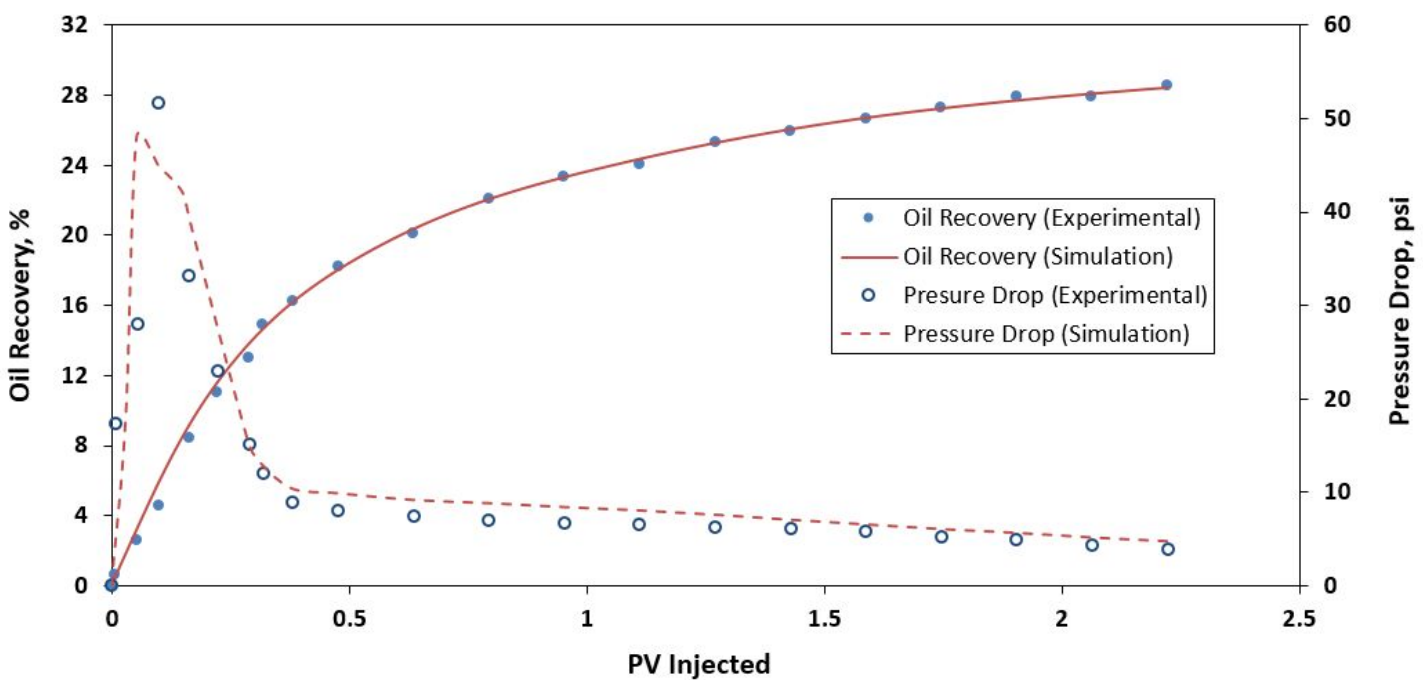

Figure 21. History match of oil recovery and pressure drop using brine-based nanofluid $(0.4$ wt. $\% \mathrm{NP}$ in $2 \% \mathrm{NaCl})$ at reservoir conditions $\left(700 \mathrm{psi} \& 150^{\circ} \mathrm{F}\right)$.

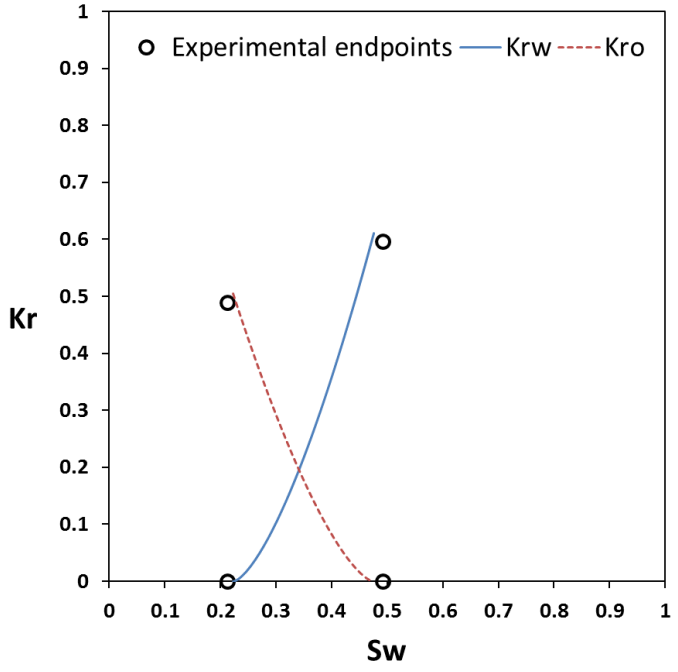

Figure 22. Relative permeability curves for coreflood using brine-based nanofluid ( $(0.4$ wt. $\% \mathrm{NP}$ in $2 \% \mathrm{NaCl})$ at reservoir conditions $\left(700\right.$ psi \& $\left.150{ }^{\circ} \mathrm{F}\right)$. 
Table 12. Initial parameters for coreflood using surfactant-based nanofluid ( 0.4 wt.\% NP + SOL 938, $2000 \mathrm{ppm})$ at reservoir conditions (700 psi \& $\left.150{ }^{\circ} \mathrm{F}\right)$.

\begin{tabular}{|ll|}
\hline Core name: & \multicolumn{2}{l|}{ Indiana Limestone-20 (12X2) } \\
Porosity: & $15.06 \quad \%$ \\
Abs. Perm: & $49.70 \quad \mathrm{md}$ \\
Pore Volume & $93 \quad \mathrm{cc}$ \\
Oil & Yates crude oil \\
Brine (NaCl) & $2 \% \quad \mathrm{wt}$ \\
Nanofluid (surfactant-based) & $0.4 \mathrm{wt} \% \mathrm{NP}+\mathrm{SOL} 938(2000 \mathrm{ppm})$ \\
Injection rate & $2 \quad \mathrm{cc} / \mathrm{min}$ \\
Oil Recovery & $51.56 \quad \%$ \\
\hline
\end{tabular}

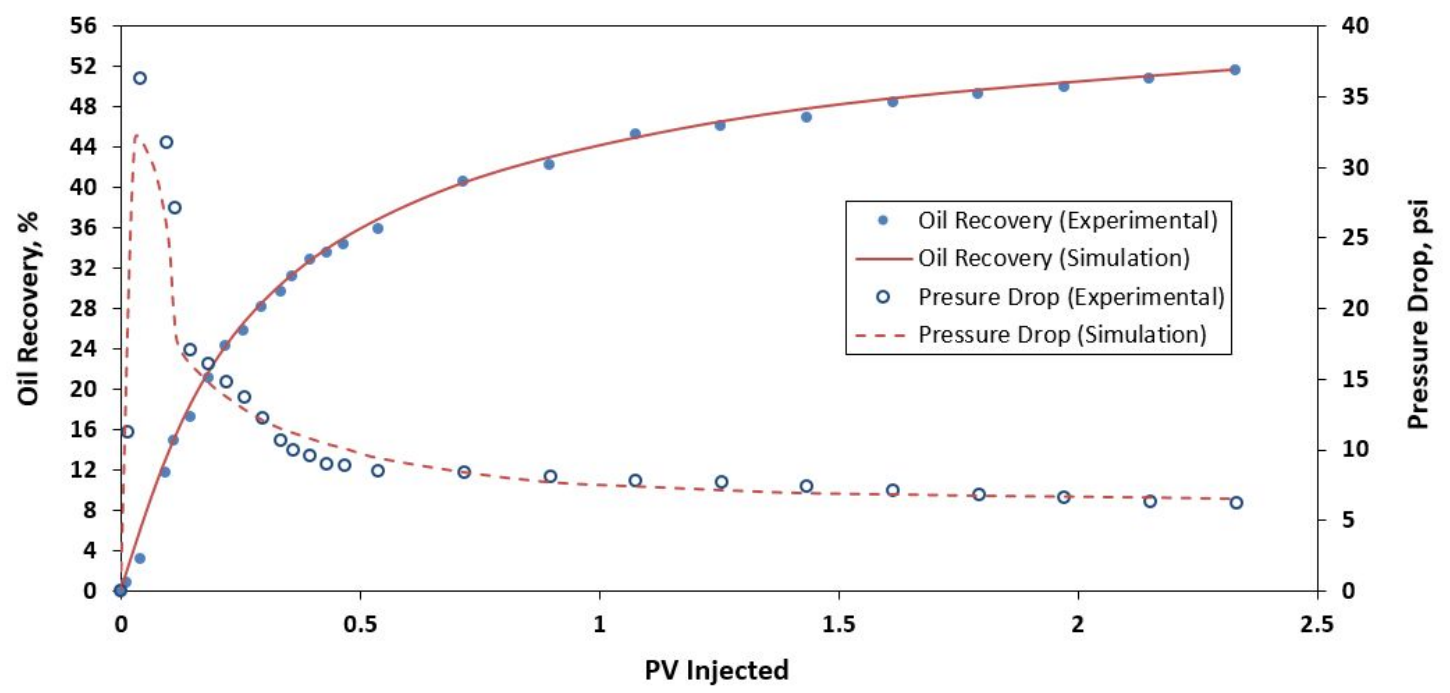

Figure 23. History match of oil recovery and pressure drop using surfactant-based nanofluid (0.4 wt.\% NP + SOL 938, $2000 \mathrm{ppm})$ at reservoir conditions (700 psi \& $\left.150{ }^{\circ} \mathrm{F}\right)$.

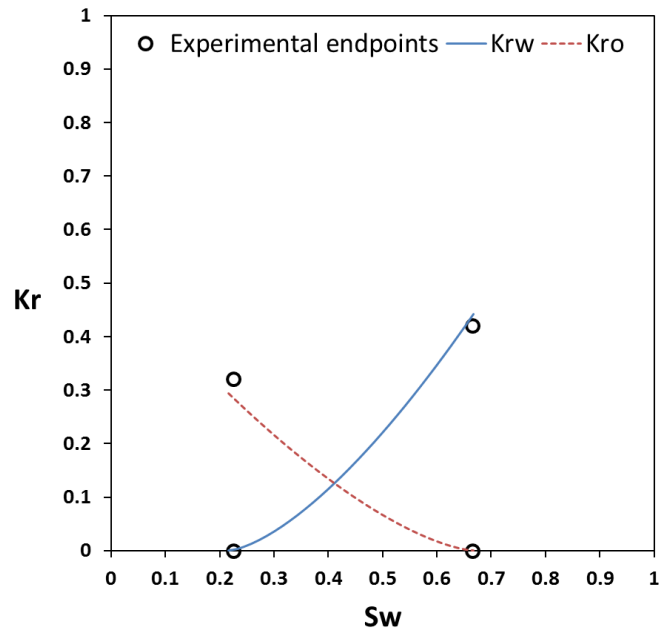

Figure 24. Relative permeability curves for coreflood using surfactant-based nanofluid (0.4 wt.\% NP + SOL 938, $2000 \mathrm{ppm})$ at reservoir conditions (700 psi \& $\left.150{ }^{\circ} \mathrm{F}\right)$. 
Table 13. Initial parameters for coreflood using surfactant-based nanofluid ( 0.4 wt.\% NP + SOL 938, $1000 \mathrm{ppm})$ at reservoir conditions (700 psi \& $\left.150{ }^{\circ} \mathrm{F}\right)$.

\begin{tabular}{|ll|}
\hline Core name: & \multicolumn{2}{l|}{ Indiana Limestone-21 (12X2) } \\
Porosity: & $17.17 \quad \%$ \\
Abs. Perm: & $62.77 \quad \mathrm{md}$ \\
Pore Volume & $106 \quad \mathrm{cc}$ \\
Oil & Yates crude oil \\
Brine (NaCl) & $2 \% \quad \mathrm{wt}$ \\
Nanofluid (surfactant-based) & $0.4 \mathrm{wt} . \% \mathrm{NP}+\mathrm{SOL} 938(1000 \mathrm{ppm})$ \\
Injection rate & $2 \quad \mathrm{cc} / \mathrm{min}$ \\
Oil Recovery & $45.07 \quad \%$ \\
\hline
\end{tabular}

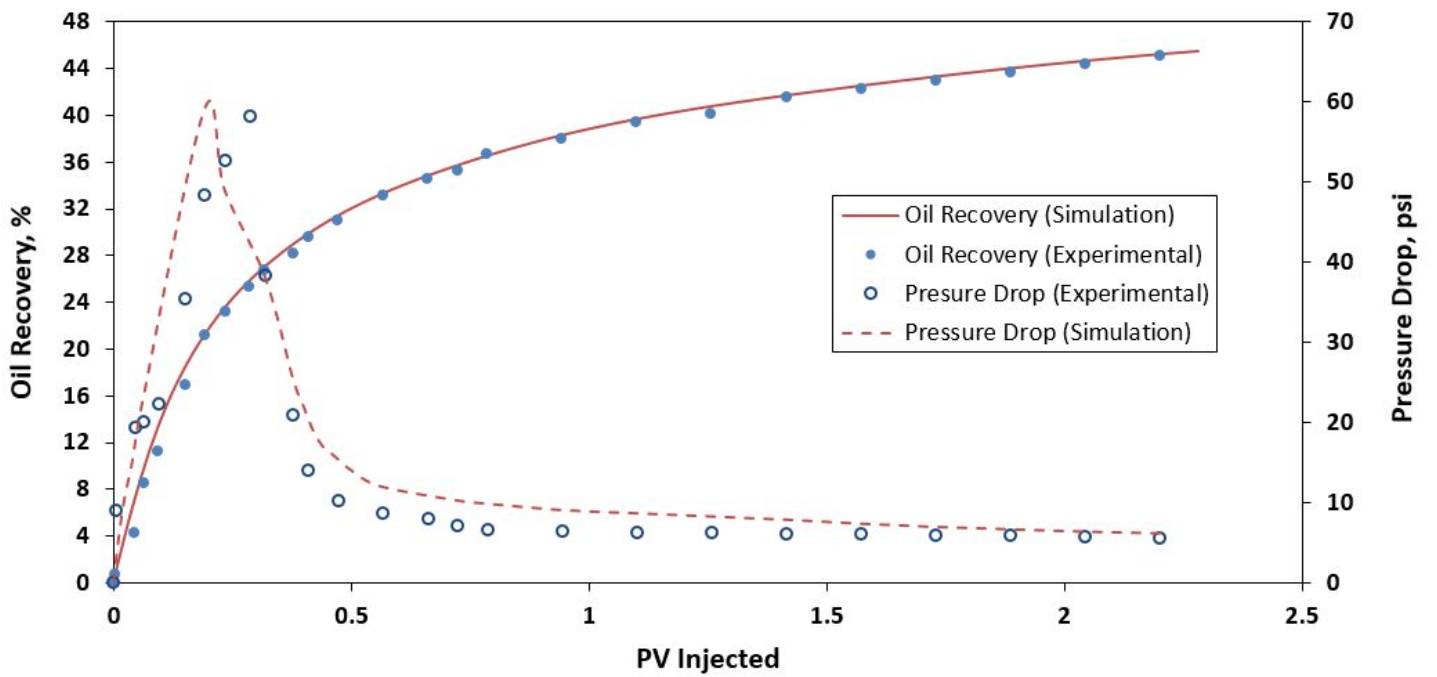

Figure 25. History match of oil recovery and pressure drop using surfactant-based nanofluid (0.4 wt.\% NP + SOL 938, $1000 \mathrm{ppm})$ at reservoir conditions (700 psi \& $\left.150{ }^{\circ} \mathrm{F}\right)$.

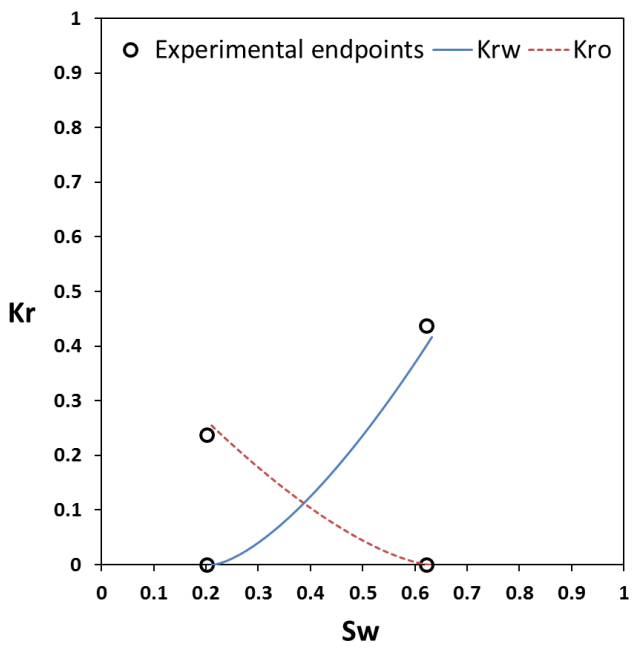

Figure 26. Relative permeability curves for coreflood using surfactant-based nanofluid $(0.4$ wt.\% NP + SOL 938, $1000 \mathrm{ppm})$ at reservoir conditions $\left(700 \mathrm{psi} \& 150{ }^{\circ} \mathrm{F}\right)$. 